\title{
ZERO ENTROPY FACTORS OF TOPOLOGICAL FLOWS
}

\author{
F. BLANCHARD AND Y. LACROIX
}

(Communicated by James E. West)

\begin{abstract}
The maximal zero entropy factor of a topological flow is defined using entropy pairs and explicitly given for some simple cartesian products. As a consequence, it is proved that only the trivial flow is disjoint from all flows whose maximal zero entropy factor is trivial.
\end{abstract}

\section{INTRODUCTION}

A topological flow is a pair $(X, T)$ where $X$ is a compact metric space and $T$ is a homeomorphism of $X$ to itself. The topological entropy of the flow $(X, T)$ is defined in [AKM]; it shall be denoted by $h(X, T)$ (if $(U, V)$ is a cover of $X$, denote by $h(U, V)$ the entropy of $(U, V))$.

Recall that a factor of the flow $(X, T)$ is a pair $(\varphi,(Y, S))$ where $(Y, S)$ is a flow and the map $\varphi:(X, T) \rightarrow(Y, S)$ is continuous, onto, and satisfies the commutation relation $\varphi \circ T=S \circ \varphi$.

In the metric theory of dynamical systems, it is known that a maximal zero entropy factor exists; it is determined by the Pinsker $\sigma$-algebra. In Topological Dynamics, however, the corresponding object has not yet been proved to exist.

The central point of this paper is the definition of the maximal zero entropy factor of a topological flow. Its construction is based on the use of entropy pairs, introduced in [B12]; it is the factor associated to the smallest closed equivalence relation collapsing all entropy pairs. This is to be compared to the way Ellis and Gottschalk proved the existence of a maximal equicontinuous factor in flows [EG].

The paper is organized as follows. In $\S 1$, we give preliminary definitions concerning entropy pairs, u.p.e. and c.p.e. flows. In $\S 2$ we examine the relation between topological factors of a flow and closed invariant equivalence relations in the product. This and entropy pairs make it possible to define the maximal zero entropy factor of the product of a flow. In $\S 3$ we give some applications, more or less close to the notion of maximal zero entropy factor. The first consists of determining the maximal zero entropy factor of the product of a flow with uniform positive entropy and a flow with zero entropy containing a dense

Received by the editors March 9, 1992.

1991 Mathematics Subject Classification. Primary 54H20.

Key words and phrases. Topological entropy, maximal zero entropy factor, disjointness.

The research of the second author was partially supported under DRET contract 901636/A000/ DRET/DS/SR. 
union of minimal subsets. The second illustrates how far the requirement of having a dense union of minimal subsets is necessary. The third is an application of the first to an example developed in [B11]. Finally, it is shown that the trivial flow is the only one disjoint from all flows having trivial maximal zero entropy factor. The appendix gives two definitions for uniform positive relative entropy and that of "entropy-flows".

The two properties of u.p.c. and c.p.e. were introduced in [B11] as an attempt to give a topological counterpart of the $K$ property of measurable dynamics: one can think of the maximal zero entropy factor as corresponding to the Pinsker $\sigma$-algebra.

But the two situations are quite different: c.p.e. is a very weak property (as shown by Proposition 3.3, or the facts about c.p.e. in [Bl1]), which is not the case of the $K$ property, though both may be formally defined in the same way; whereas u.p.e., a perfectly natural definition in view of the properties of entropy pairs, seems not to correspond to any metric property.

\section{Preliminaries}

Most of the following definitions and statements are taken from [B12].

- A standard cover of $X$ is a pair $(U, V)$ of nondense open sets of $X$, covering $X$.

- If $x, x^{\prime} \in X$, and $(U, V)$ is a standard cover of $X$, we shall say that $x$ and $x^{\prime}$ are distinguished by the cover $(U, V)$ if and only if $x \in \operatorname{Int}\left(U^{c}\right)$ and $x^{\prime} \in \operatorname{Int}\left(V^{c}\right)$.

- A pair of points $\left(x, x^{\prime}\right)$ in $X^{2}$ is called an entropy pair if and only if any standard cover distinguishing $x$ and $x^{\prime}$ has positive entropy.

Let us define the set

$$
E(X, T)=\left\{\left(x, x^{\prime}\right) \in X^{2},\left(x, x^{\prime}\right) \text { is an entropy pair }\right\} .
$$

Denoting by $\Delta$ the diagonal subset of $X^{2}$, the set $E(X, T) \cup \Delta$ is a closed $(T \times T)$-invariant subset of $X^{2}$.

A flow $(X, T)$ has uniform positive entropy (abbreviated as "has u.p.e.") if and only if any standard cover of $(X, T)$ has strictly positive entropy (in [B11] this property was shown to imply weak mixing).

One easily checks that $(X, T)$ has u.p.e. if and only if $E(X, T) \cup \Delta=X^{2}$. One proves that $h(X, T)>0$ if and only if $E(X, T) \neq \varnothing$.

A flow $(X, T)$ is said to have c.p.e. (completely positive entropy) flow if and only if all its nontrivial factors have positive entropy.

If $\varphi:(X, T) \rightarrow(Y, S)$ is a factor, then the two following properties hold.

Property (a). If $\left(x, x^{\prime}\right) \in E(X, T)$ and $\varphi(x) \neq \varphi\left(x^{\prime}\right)$, then $\left(\varphi(x), \varphi\left(x^{\prime}\right)\right) \in$ $E(Y, S)$.

Property (b). If $\left(y, y^{\prime}\right) \in E(Y, S), \exists x, x^{\prime} \in X, \varphi(x)=y, \varphi\left(x^{\prime}\right)=y^{\prime}$, and $\left(x, x^{\prime}\right) \in E(X, T)$.

The following is another elementary property of entropy pairs, which proves useful in the sequel.

Proposition 1.1. Suppose $X^{\prime}$ is a closed T-invariant subset of $(X, T)$. Then if $\left(x, x^{\prime}\right)$ is an entropy pair of $\left(X^{\prime}, T\right)$, it is also an entropy pair of $(X, T)$. 


\section{Closed and equivalence $(T \times T)$-INVARiant Relations}

Let $(X, T)$ be a flow and $G \subset X \times X$ a closed $(T \times T)$-invariant equivalence relation. Let $p_{G}: X \rightarrow X /{ }_{G}$ denote the quotient map.

Assume the metric $d(\cdot, \cdot)$ defines the compact topology on $X$. For $x \in X$ let $(x)$ be the closed set $\{y \in X \mid(x, y) \in G\}$; endow $X / G$ with the Hausdorff metric, which induces the usual quotient topology on this set; furthermore, $p_{G}$ is continuous and $T$ induces a homeomorphism $T_{G}$ on $X /{ }_{G}$ by the formula $T_{G}((x)):=p_{G}(T x)$. These constructions lead to the following commutative diagram:

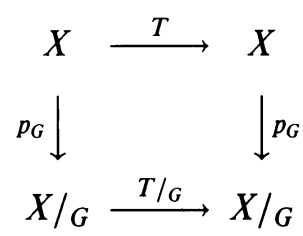

Conversely, any factor $\varphi:(X, T) \rightarrow(Y, S)$ determines a closed $(T \times T)$ invariant equivalence relation $G_{\varphi} \subset X \times X$ :

$$
\left(\varphi(x)=\varphi\left(x^{\prime}\right)\right) \Leftrightarrow\left(\left(x, x^{\prime}\right) \in G_{\varphi}\right),
$$

and $\left(X / G_{\varphi}, T / G_{\varphi}\right)$ is topologically conjugate to $(Y, S)$ via the map $\varphi^{\prime}$ :

$$
\varphi^{\prime}\left(p_{G_{\varphi}}(x)\right):=\varphi(x), \quad x \in X .
$$

Thus, factors of $(X, T)$ are in a one-to-one correspondence with closed $(T \times T)$ invariant equivalence relations in $X \times X$.

Assume $G$ and $G^{\prime}$ are two such relations and furthermore $G^{\prime} \subset G$; then one can define the map $p_{G, G^{\prime}}: X / G_{G^{\prime}} \rightarrow X / G$ by the formula

$$
p_{G, G^{\prime}}\left(p_{G^{\prime}}(x)\right):=p_{G}(x), \quad x \in X .
$$

Then we have the following commutative diagram:

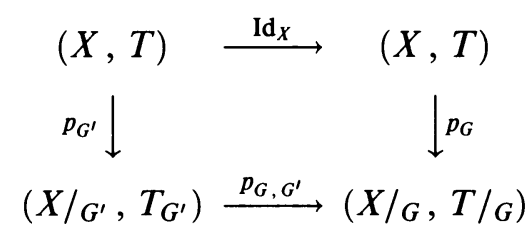

Reciprocally, if

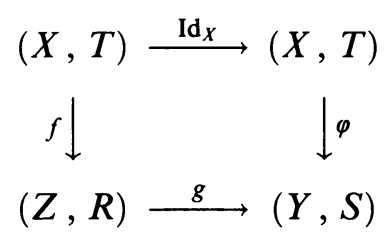

is a commutative diagram of factors, then $G_{f} \subset G_{\varphi}$.

Next, if $R$ is a subset of $X \times X$ and $\mathscr{F}(R)$ is the set of closed $(T \times T)$ invariant equivalence relations containing $R, \mathscr{F}(R)$ is not empty. Moreover, since $\bigcap_{G \in \mathscr{F}(R)} G \in \mathscr{F}(R), \mathscr{F}(R)$ has, once it is partially ordered with inclusion, a minimal element, the intersection mentioned above. Let

$$
G(R)=\bigcap_{G \in \mathscr{F}(R)} G \in \mathscr{F}(R) .
$$


Define

$$
G_{0}(X, T)=G(E(X, T)) .
$$

For a flow $(X, T)$, let $\left(X_{0}, T_{0}\right):=\left(X / G_{0}(X, T), T / G_{0}(X, T)\right)$ and $p_{0}=p_{G_{0}(X, T)}$. Theorem 2.1. Let $\varphi:(X, T) \rightarrow(Y, S)$ be a factor map. Then $h(Y, X)=0$ if and only if there exists a factor map $f:\left(X_{0}, T_{0}\right) \rightarrow(Y, S)$ such that the following diagram is commutative:

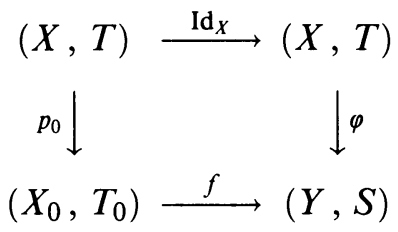

Proof. Assume $h(Y, S)=0$. Then by definition of entropy pairs $E(Y, S)=$ $\varnothing$. By Property (a) this means that if $\left(x, x^{\prime}\right) \in E(X, T)$ one must have $\varphi(x)=\varphi\left(x^{\prime}\right)$; hence, $G_{0}(X, T) \subset G_{\varphi}$, and from the remarks above there follows existence of a map $f$ with the suitable properties.

Conversely, suppose $(Y, S)$ is a factor of $\left(X_{0}, T_{0}\right)$. Indeed, if this were false $E\left(X_{0}, T_{0}\right)$ would contain some pair $\left(z, z^{\prime}\right)$ with $z \neq z^{\prime}$; by Property (b) this means some entropy pair of $(X, T)$ would have image $\left(z, z^{\prime}\right)$ under $p_{0}$, thus not being collapsed, which contradicts the definition of $p_{0}$. Therefore $h(Y, S)$ is also 0 .

Definition 2.1. $\left(X_{0}, T_{0}\right)$ is called the maximal zero entropy factor of $(X, T)$.

\section{Applications}

It is now clear that the determination of the maximal zero entropy factor of a flow relies on the localisation of its entropy pairs.

Application 1. Let $(X, T)$ have u.p.e. and $h(Y, S)=0$. The expected is that the maximal zero entropy factor of the product flow $(X \times Y, T \times S)$ is $(Y, S)$. Surprisingly, this is not always the case and requires an additional condition. Recall that a minimal flow is one with no proper closed invariant subset.

Proposition 3.1. Let $(X, T)$ have u.p.e., $h(Y, S)=0$, and assume $(Y, S)$ contains a dense union of minimal sets. Then

$$
E(X \times Y, T \times S) \cup \Delta_{X \times Y}=\left\{\left((x, y),\left(x^{\prime}, y\right)\right) ; x, x^{\prime} \in X, y \in Y\right\},
$$

and the maximal zero entropy factor of $(X \times Y, T \times S)$ is $(Y, S)$.

Proof. First suppose $h(Y, S)=0$ only. The projections of $(X \times Y, T \times S)$ on $(X, T)$ and $(Y, S)$ are factors. Suppose

$$
\left((x, y),\left(x^{\prime}, y^{\prime}\right)\right) \in E(X \times Y, T \times S) \cup \Delta .
$$

Then, by Property (a), either $\left(y, y^{\prime}\right) \in E(Y, S)$ or $y=y^{\prime}$. Since $h(Y, S)=0$, $E(Y, S)=\varnothing$, and we obtain $E(X \times Y, T \times S) \cup \Delta_{X \times Y} \subset\left\{\left((x, y),\left(x^{\prime}, y\right)\right)\right.$; $\left.x, x^{\prime} \in X, y \in Y\right\}$.

In order to prove the reverse inclusion, let us first use the u.p.e. assumption on $(X, T)$. As u.p.e. implies weak mixing [B11, Proposition 2] there is some pair $\left(x, x^{\prime}\right) \in X^{2}$ having dense orbit under $T \times T$. It cannot belong to the diagonal, so $x \neq x^{\prime}$ and $\left(x, x^{\prime}\right)$ is an entropy pair of $(X, T)$. Thus, by Property (b), 
there is $y \in Y$ such that $\left((x, y),\left(x^{\prime}, y\right)\right) \in E(X \times Y, T \times S)$. Now, $T \times \operatorname{Id}_{Y}$ is an automorphism of the product flow; by Property (a), all points in the orbit of $\left((x, y),\left(x^{\prime}, y\right)\right)$ under $T \times \operatorname{Id}_{Y}$ belong to $E(X \times Y, T \times S) \cup \Delta_{X \times Y}$. On account of the choice of $\left(x, x^{\prime}\right)$, their closure is all of $(X \times\{y\}, X \times\{y\})$, so $(X \times\{y\}, X \times\{y\}) \subset E(X \times Y, T \times S) \cup \Delta_{X \times Y}$.

There remains to prove this is true for any $y \in Y:$ this is where the additional assumption on $(Y, S)$ comes in. When $(Y, S)$ is minimal, it is sufficient to apply Property (a) to the action of the endomorphism $\operatorname{Id}_{X} \times S$ on $(X \times\{y\}$, $X \times\{y\})$ and then closedness of $E(X \times Y, T \times S) \cup \Delta_{X \times Y}$. If $(Y, S)$ merely contains a dense union of minimal $\left(Y_{i}, S\right), i \in I$, we may apply the last result to each $\left(X \times Y_{i}, T \times S\right), i \in I$, and then Proposition 1.1.

$E(X \times Y, T \times S) \cup \Delta_{X \times Y}$ is obviously the graph of an equivalence relation. Call $\varphi$ the associated homomorphism; $\varphi$ identifies points having the same projection on $Y$ and never identifies points having projections on $Y$. This means $\varphi(X \times Y, T \times S)=(Y, S)$.

When the union of minimal subsets does not exist, the conclusion of Proposition 3.1 does not hold. The following example illustrates this claim.

Application 2. Let $(X, T)$ have u.p.e., and let $Y=\mathbf{N} \cup\{\infty\}$ be the Alexandroff compactification of $\mathbf{N}$. Let $S$ be the translation by 1 on $Y$, having $\infty$ as unique fixed point. $(Y, S)$ is not transitive, and $h(Y, S)=0$ as will be seen below. The set $X \times\{\infty\}$ is conjugate to $X$ inside $(X \times Y, T \times S)$, so its cartesian square is contained in $E(X \times Y, T \times S) \cup \Delta$ by Proposition 1.1.

Suppose $(U, V)$ is a standard cover of $X$; if $i \in \mathbf{N}, U^{c} \times\{i\}$ and $V^{c} \times\{i\}$ are two closed sets of $X \times Y$ with nonempty interior so that $U^{\prime}:=\left(U^{c} \times\{i\}\right)^{c}$ and $V^{\prime}:=\left(V^{c} \times\{i\}\right)^{c}$ form a standard cover $\mathscr{R}$ of $X \times Y$. This cover has entropy zero, merely on account of the second coordinate; for $x \in X, n \in \mathbf{N}$, $(T \times S)^{k}(x, n) \in U^{\prime} \cap V^{\prime}$ whenever $n+k \neq i$; if $k=i-n$ then $(x, n) \in$ $(T \times S)^{n-i} U^{\prime}$ or $(x, n) \in(T \times S)^{n-i} U^{\prime}$ according to the position of $x$ in $X$. Now choose some $p$ and consider cover $\mathscr{R}_{p}=\bigvee_{0 \leq i<p} T^{-i} \mathscr{R}$. All points whose orbit from 0 to $p-1$ never reaches $V^{c} \times\{i\}, i=0, \ldots, p-1$, are in the set $U^{\prime} \cap(T \times S)^{-1} U^{\prime} \cap \cdots \cap(T \times S)^{-p+1} U^{\prime}$; the others belong to one of the sets $U^{\prime} \cap \cdots \cap(T \times S)^{-i+1} U^{\prime} \cap(T \times S)^{-i} V^{\prime} \cap(T \times S)^{-i-1} U^{\prime} \cap \cdots \cap(T \times S)^{-p+1} U^{\prime}$. We have thus found a subcover of $\mathscr{R}_{p}$ with cardinality $p+1$, and $h(\mathscr{R}, T \times S)=0$. For $x \neq x^{\prime}, \mathscr{R}$ can be chosen so as to distinguish $(x, i)$ and $\left(x^{\prime}, i\right)$, which implies there is no entropy pair of the form $\left((x, i),\left(x^{\prime}, i\right)\right), i \in \mathbf{N}, x \neq x^{\prime}$. As was remarked in the proof of Proposition 3.1 no pair of the form $\left((x, i),\left(x, i^{\prime}\right)\right)$, $i \neq i^{\prime}$, may be in $E(X \times Y, T \times S)$. Therefore, $E(X \times Y, T \times S) \cup \Delta=$ $(X \times\{\infty\})^{2}$.

$(X \times\{\infty\})^{2} \cup \Delta$ is the graph of an equivalence relation. The associated homomorphism $\varphi$ identifies all points $(x, \infty)$ and them only. In the flow $(X \times Y, T \times S)$ the maximal zero entropy factor is therefore much bigger than $(Y, S)$.

Application 3. Here is an application of Proposition 3.1.

Let $Y$ be a subshift of $A^{\mathbf{Z}}, 0 \notin A$, and let $\varphi:\{0,1\}^{\mathbf{Z}} \times Y \rightarrow(A \cup\{0\})^{\mathbf{Z}}$ be defined by the alphabetic map $\bar{\varphi}:\{0,1\} \times A \rightarrow A \cup\{0\}: \bar{\varphi}(1, a)=a$, $\bar{\varphi}(0, a)=0, a \in A$. Let $Z=\varphi\left(\{0,1\}^{Z} \times Y\right)$.

It was shown in [Bl1, Proposition 10] that $Z$ has c.p.e. (i.e., its maximal 
zero entropy factor is trivial) whenever $Y$ is minimal, but also that it has not got u.p.e. when $h(Y)=0$. This is obvious when one looks at entropy pairs. If $(Y, \sigma)$ is minimal with entropy 0 , since $\{0,1\}^{\mathbf{Z}}$ has u.p.e., by Proposition 3.1., $E(X \times Y, \sigma \times \sigma) \cup \Delta=\left\{\left((x, y),\left(x^{\prime}, y\right)\right) ; x, x^{\prime} \in X, y \in Y\right\}$. Call $z_{0}$ the fixed point on letter 0 in $Z$ and $x_{0}, x_{1}$ the fixed points on letters 0 and 1 in $\{0,1\}^{\mathbf{Z}}$. Given $z \in Z$ one easily constructs a unique point $x \in\{0,1\}^{\mathbf{Z}}$ such that $z=\varphi(x, y)$ by replacing all symbols of $A$ in $\mathbf{Z}$ by 1 's, but $y$ is not generally unique; nevertheless, $Z$ contains a copy of $Y: \varphi\left(x_{1}, y\right)=y$, and $\left(x_{1}, y\right)$ is the only point with image $y$ under $\varphi$. As $\left(\left(x_{1}, y\right),\left(x_{1}, y^{\prime}\right)\right)$ never belongs to $E\left((X \times Y, \sigma \times \sigma)\right.$, this implies $\left(y, y^{\prime}\right) \notin E(Z)$ for $y \neq y^{\prime}$; therefore, $Z$ does not have u.p.e.

Nevertheless, its maximal zero entropy factor is trivial. For any $z \in \varphi(x, y)$ $\neq z_{0}$, one has $\left(z_{0}, z\right)=\left(\varphi\left(x_{0}, y\right), \varphi(x, y)\right) \in E(X \times Y, \sigma \times \sigma)$. Hence, from Properties (a) and (b), $\left(z_{0}, z\right) \in E(Z, \sigma)$. The smallest equivalence relation whose graph contains $E(Z, \sigma)$ identifies all points with $z_{0}$, the associated homomorphism thus having trivial image.

Application 4. The proof of the next statement combines ideas from the proof of Proposition 3.1 and a construction generalizing that of Example 3.

Two flows $(X, T)$ and $(Y, S)$ are said to be disjoint if there exists no proper closed $(T \times S)$-invariant subset of $(X \times Y, T \times S)$ having projections $X$ and $Y$ on the two coordinates. For two flows to be disjoint, one of them at least must be minimal $[\mathrm{F}]$.

Proposition 4.3. The set of flows disjoint from all c.p.e. flows is reduced to the trivial flow.

Proof. Flows disjoint from all c.p.e. flows are necessarily minimal, because some c.p.e. flows are not (the full shift for example). It is therefore sufficient to prove that, for any minimal flow, one can construct a nondisjoint c.p.e. flow.

Let $(X, T)$ have u.p.e. and contain a fixed point $t$ (take, for instance, a full shift) and $(Y, S)$ be nontrivial and minimal. For some metric $d$ associated with the topology of $X$ define the continuous function $f$ from $X$ to $[0,1]: f(x)=d(t, x)$; one has $\min _{x} f(x)=f(t)=0$, and without loss of generality one may assume $\max _{x} d(t, x)=1$. Choose some point $u \in Y$ and some $a, 0<a<1$, and put $g(y)=a+d(u, y) ; g$ is continuous; one has $\min _{y} g(y)=g(u)=a, \max _{x} g(y)=b>a$ since $Y$ is not a singleton. Denote by $\omega_{n}$ the coordinate of the doubly infinite sequence $\omega$ at time $n$. Functions $f$ and $g$ allow us to define a homomorphism $\varphi$ from $(X \times Y, T \times S)$ onto some closed shift-invariant subset $Z$ of $[0, b]^{\mathrm{Z}}$, endowed with the shift $\sigma$, by the formula $(\varphi(x, y))_{n}=f\left(T^{n} x\right) \cdot g\left(S^{n} y\right), n \in \mathbf{Z}:$ the fixed image $s=\varphi(t, y)$ for any $y \in Y$ is the fixed point on real number 0 .

Let us prove $Z$ has c.p.e. For this we show that whatever $z \in Z, z \neq s$, $(s, z) \in E(Z, \sigma)$. Since $z \in Z$ there is a pair $\left(x^{\prime}, y^{\prime}\right)$ such that $z=\varphi\left(x^{\prime}, y^{\prime}\right)$, so $(s, z)=\left(\varphi(t, y), \varphi\left(x^{\prime}, y^{\prime}\right)\right)$ for any $y$ and for some $x^{\prime} \neq t$. As $(X, T)$ has u.p.e., one has $\left(t, x^{\prime}\right) \in E(X, T)$; therefore, by Property (b) there are $y_{1}, y_{1}^{\prime} \in Y$ such that $\left(\left(t, y_{1}\right),\left(x^{\prime}, y_{1}^{\prime}\right)\right) \in E(X \times Y, T \times S)$. Now since $Y$ is minimal, the orbit of $y_{1}^{\prime}$ under $S$ is dense in $Y$; Id $\times S$ is a homomorphism of $(X \times Y, T \times S)$, so by Property (a) and closedness of $E(X \times Y, T \times S) \cup \Delta$, there is $y_{2} \in Y$ such that $\left(\left(t, y_{2}\right),\left(x^{\prime}, y^{\prime}\right)\right) \in E(X \times Y, T \times S)$; now this is enough: by Property (a) again $\left(\varphi\left(t, y_{2}\right), \varphi\left(x^{\prime}, y^{\prime}\right)\right)=(s, z) \in E(Z, \sigma)$. To 
finish this part of the proof remark that since all points $z \neq s$ in $Z$ form an entropy pair with $s$, the smallest equivalence relation saturating $E(Z, \sigma)$ is trivial; so is the maximal zero entropy factor of $(Z, \sigma)$.

Finally $(Z, \sigma)$ is not disjoint from $(Y, S)$; the set

$$
F=\{(z, y) \in Z \times Y ; \exists x \in X: \varphi(x, y)=z\} \subset Z \times Y
$$

is obviously closed and invariant by $\sigma \times S$; it has image $Z$ under projection on the first coordinate, since the defining condition rests on $y$ alone, and image $Y$ under projection on the second by minimality. $F$ is also a proper subset of $Z \times Y$ : there exists $z \in \mathbf{Z}$ such that $z_{0}=b>a$; then for any $x \in X$, $f(x) \cdot g(u) \leq a<b$, so that $(z, u) \notin F$.

\section{APPENDIX}

The notion of relative $K$-systems has proved useful in Ergodic Theory; so might that of relative u.p.e. for flows. Here are two possible definitions.

Define the relation

$$
\left(x \mathscr{R}_{\varphi} x^{\prime}\right) \Leftrightarrow\left(\left(\varphi(x)=\varphi\left(x^{\prime}\right)\right) \text { or }\left(\varphi(x) \neq \varphi\left(x^{\prime}\right) \text { and }\left(\varphi(x), \varphi\left(x^{\prime}\right)\right) \in E(Y, S)\right)\right) \text {. }
$$

Definition A.1. Let $\varphi:(X, T) \rightarrow(Y, S)$ be a factor map. We shall say that $\varphi:(X, T) \rightarrow(Y, S)$ is a factor of uniform positive relative entropy (and abbreviate " $\varphi$ is an f.u.p.r.e.") if and only if the following equivalence relation holds:

$$
\left(\left(x \mathscr{R}_{\varphi} x^{\prime}\right) \text { and }\left(x \neq x^{\prime}\right)\right) \Leftrightarrow\left(\left(x, x^{\prime}\right) \in E(X, T)\right) .
$$

In other words, if we denote by the same symbol $\mathscr{R}_{\varphi}$ the graph of $\mathscr{R}_{\varphi}$ in $X \times X$, the factor $\varphi$ is an f.u.p.r.e. of $(X, T)$ if and only if

$$
\mathscr{R}_{\varphi} \backslash \Delta \subseteq E(X, T) .
$$

With the first definition, we have the following.

Proposition A.1. Let $\varphi:(X, T) \rightarrow(Y, S)$ be an f.u.p.r.e. Then $p_{0}$ factors through $\varphi$.

Proof. It is enough to show that when $\varphi$ is an f.u.p.r.e., $G_{\varphi} \subset E(X, T) \cup \Delta$. By definition of an f.u.p.r.e., this condition is fulfilled.

One can prove the following:

Proposition A.2. Assume the following diagram of topological factors is commutative:

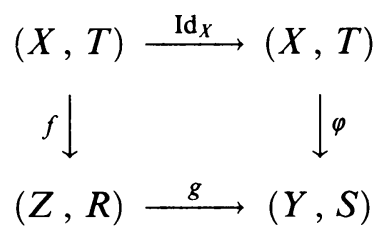

Then $\varphi$ is an f.u.p.r.e. if and only if $f$ and $g$ both are.

Definition A.2. A flow $(X, T)$ shall be called an entropy flow, say " $E$-flow", if and only if $E(X, T) \cup \Delta$ is an equivalence relation. 
Property A.1. An E-flow is an f.u.p.r.e. with respect to its maximal zero entropy factor.

Proposition 3.1 provides some (rather naive) examples of f.u.p.r.e. and entropy flows.

\section{ACKNOWLEDGMENT}

We would like to express to Marius Lemañczyk our special thanks for pointing out many errors and making useful suggestions.

\section{REFERENCES}

[AKM] R. L. Adler, A. G. Konheim, and M. H. McAndrew, Topological entropy, Trans. Amer. Math. Soc. 114 (1965), 309-319.

[Bl1] F. Blanchard, Full positive topological entropy and topological mixing, Symbolic Dynamics and Applications (in honor of R. L. Adler), Contemp. Math., Amer. Math. Soc., Providence, RI (to appear).

[B12] _ A disjointness theorem involving topological entropy, preprint, 1991.

[EG] R. Eilis and W. H. Gottschalk, Homomorphisms of transformation groups, Trans. Amer. Math. Soc. 94 (1960), 258-271.

[F] H. Furstenberg, Disjointness in ergodic theory, minimal sets, and a problem in diophantine approximation, Math. Systems Theory 1 (1967), 1-49.

Université de Provence, CNRS URa 225, Case X, 3 place V. Hugo, 13331 Marseille Cedex 3, France

Current address, F. Blanchard: LMD-CNRS, case 930, 163 avenue de Luminy, 13288 Marseille Cedex 09, France

E-mail address: blanchar@lmd.univ-mrs.fr

Université de Provence, UfR-MiM, Case 92, 3 place V. Hugo, 13331 Marseille Cedex

3, FRANCE

E-mail address: lacroix@kelenn-gw.cicb.fr 